\title{
FUSION OF MAGNETIC RESONANCE AND ULTRASOUND IMAGES: A PRELIMINARY STUDY ON SIMULATED DATA
}

\author{
Oumaima El Mansouri ${ }^{1,2}$, Adrian Basarab ${ }^{2}$, Fabien Vidal ${ }^{2,3}$ Denis Kouamé ${ }^{2}$, Jean-Yves Tourneret ${ }^{1}$, \\ ${ }^{1}$ University of Toulouse, IRIT/INP-ENSEEIHT, 31071 Toulouse Cedex 7, France \\ ${ }^{2}$ University of Toulouse, IRIT, CNRS UMR 5505, Université Paul Sabatier, Toulouse, France \\ ${ }^{3}$ CHU Toulouse, Obstetrics Gynecology Department, Paule de viguier Hospital Toulouse F-31059, France \\ \{el-mansouri, vidal, basarab, kouame, tourneret\}@irit.fr
}

\begin{abstract}
We propose a new fusion method for magnetic resonance imaging (MRI) and ultrasound (US) data combining two inverse problems: MRI reconstruction using super-resolution and US image despeckling, using a model relating the two modalities through an unknown polynomial function. We demonstrate the accuracy of the proposed fusion algorithm by quantitative and qualitative evaluation using synthetic data. The resulting fused image is shown to have an improved signal to noise ratio and spatial resolution compared to the native MRI and US images.
\end{abstract}

Index Terms - Fusion MRI and ultrasound, super-resolution, despeckling, alternating direction method of multipliers, total variation regularization.

\section{INTRODUCTION}

Image fusion can be generally defined as "gathering all the important information from multiple images, and including them into a fewer number of images" [1], e.g., into one single image. This single image is usually more informative and accurate than the images before fusion and contains all the necessary information for the application of interest" [1]. Fusion of medical images is an important and challenging research field facilitating medical diagnosis and decisions in number of clinical studies. Existing medical image fusion include positron emission tomography (PET) and magnetic resonance imaging (MRI) [2], or gammagraphy and ultrasound (US) imaging [3]. However, to the best of our knowledge, the fusion of MR and US images has been only considered in the literature from the perspective of image registration [4] and not as an image fusion problem, which is precisely the aim of this work.

MR and US images are two different medical modalities having their own advantages and drawbacks. For example, for precise localization and characterization of small lesions such as those occurring within endomotriosis disease, US images allow their depth of infiltration to be evaluated using transvaginal or transrectal probes. However, these images are characterized by a low signal to noise ratio and a limited field of view. On the other hand, MR images provide a wider field of view of the patient anatomy with a good signal to noise ratio. However, small lesions cannot be detected with MRI because of its limited spatial resolution of the order of 1 to $2 \mathrm{~mm}$. The complementary properties of US and MR images explain why medical doctors currently need both modalities for the diagnosis of many diseases. The final aim of this work is to perform MR and US image fusion for endometriosis surgery, that requires to localize and to border the endometriosis [5] and to know precisely its depth of infiltration, especially if the endometrial implant is inside or under an organ. As a consequence, endometriosis diagnosis needs both MRI and US modalities. Being able to fuse MRI and US images would help the surgeon to overcome the dissimilarities between these two kinds of images and to improve the medical decision.

This paper studies a new fusion method for MR and US images. The proposed method is tested on simulated data with available ground truth allowing quantitative evaluation of the fused image. The remainder of the paper is organized as follows. Section 2 presents a statistical model for MRI and US image fusion including a linear relation between these two modalities. Section 3 introduces the MRI/US fusion algorithm investigated in this work. Simulation results are presented in Section 4 whereas conclusions and perspectives are reported in Section 5.

\section{A NEW STATISTICAL MODEL FOR THE FUSION OF MRI AND US IMAGES}

This work assumes that the MR and US images to be fused have been registered. Thus, registration (e.g., [4]) is considered herein as a pre-processing step and the possible registration errors are ignored hereafter. Note that despite this hypothesis, the fusion task is still challenging because of the differences in resolution, contrast and noise between the two imaging modalities.

\subsection{Observation models}

In several applications including gynecology (considered in this study), MRI has the advantage of acquiring images with a large field of view, at the expense of a relatively low spatial resolution, of the order of 1 to $2 \mathrm{~mm}$. In contrast to MRI, depending on the choice of the probe's central frequency, US imaging can offer well-resolved images but contaminated by a high level of speckle noise and with a reduced field of view. Based on these observations, many existing works aimed at improving independently the quality of MR and US images. In the case of MRI, the loss of resolution is classically modelled by a downsampling operation and a low pass filter, e.g., see [6]. In US imaging, speckle noise is considered as additive when considering the log-compressed envelope mode (also called B-mode) with a log-Rayleigh distribution [7,8]. These observations lead to the following statistical models

$$
\begin{aligned}
\mathbf{y}_{\mathrm{m}} & =\mathbf{S H x}_{\mathrm{m}}+\mathbf{n}_{\mathrm{m}} \\
\mathbf{y}_{\mathrm{u}} & =\mathbf{x}_{\mathrm{u}}+\mathbf{n}_{\mathrm{u}}
\end{aligned}
$$

where $\mathbf{x}_{\mathrm{m}} \in \mathbb{R}^{N}$ is the non-observable high-resolution MR image, $\mathbf{y}_{\mathrm{m}} \in \mathbb{R}^{M}$ is the low-resolution MR image, $\mathbf{n}_{\mathrm{m}} \in \mathbb{R}^{N}$ is an independent identically distributed i.i.d. additive white Gaussian noise with variance $\sigma_{\mathrm{m}}^{2}, \mathbf{H} \in \mathbb{R}^{N \times N}$ is a block circulant with circulant 
blocks matrix (modelling the blurring effect of the PSF with circulant boundary conditions), $\mathbf{S} \in \mathbb{R}^{M \times N}$ (with $N=d^{2} M$ ) is a decimation operator with a decimation factor $d, \mathbf{y}_{\mathrm{u}} \in \mathbb{R}^{N}$ is the B-mode US image, $\mathbf{x}_{\mathrm{u}} \in \mathbb{R}^{N}$ is the noise-free US image and $\mathbf{n}_{\mathrm{u}} \in \mathbb{R}^{N}$ is an i.i.d. additive log-Rayleigh noise sequence with localization parameter $\gamma$. Note that in (1) all the vectors are obtained by stacking the corresponding images into column vectors in a lexicographic order. Note also that we suppose that $\mathbf{x}_{\mathrm{m}}$ and $\mathbf{x}_{\mathrm{u}}$ have the same spatial sampling. In practical situations, this can be obtained by adjusting the decimation factor $d$ in the MRI model, so that pixels in the superresolved MR and US images have the same size.

\subsection{Relation between US and MR images}

MR and US imaging technologies exploit different physical phenomena. Consequently, when imaging the same tissues and even in the virtual case where the acquisition is perfect, they do not provide the same pixel amplitude. Thus, images $\mathbf{x}_{\mathrm{m}}$ and $\mathbf{x}_{\mathrm{u}}$ in (1) are not identical, even if they represent the same imaged tissues. For solving the fusion task, we propose to link these two images by a parametric model. In this work, we adopt the model proposed in [9] for MR/US image registration. This model originates from the observation that US images are formed due to the interfaces between anatomical structures having different acoustic impedances, which are related with the gradient of the MR image, i.e.,

$$
\mathbf{x}_{\mathrm{u}}=f\left(\mathbf{x}_{\mathrm{m}},\left\|\nabla \mathbf{x}_{\mathrm{m}}\right\|_{\mathbf{2}}\right)
$$

where $f: \mathbb{R}^{\mathbb{N}} \times \mathbb{R}^{\mathbb{N}} \rightarrow \mathbb{R}^{\mathbb{N}}$ is an unknown function and $\left\|\nabla \mathbf{x}_{\mathrm{m}}\right\|_{2} \in$ $\mathbb{R}^{\mathbb{N}}$ is the $\ell_{2}$-norm of the local gradient of $\mathbf{x}_{\mathrm{m}}$, whose $i$ th component (corresponding to pixel $i$ ) is defined as

$$
\left\|\nabla \mathbf{x}_{\mathrm{m}}\right\|_{\mathrm{i}}=\sqrt{\left(\mathbf{D}_{\mathrm{h}} \mathbf{x}_{\mathrm{m}}\right)_{\mathrm{i}}^{2}+\left(\mathbf{D}_{\mathrm{v}} \mathbf{x}_{\mathrm{m}}\right)_{\mathrm{i}}^{2}}
$$

where $\mathbf{D}_{\mathrm{h}}$ and $\mathbf{D}_{\mathrm{h}}$ are two matrices accounting for horizontal and vertical discrete differentiation.

Eq. (2) shows that the relation between MR and US images is governed by the choice of an appropriation function $f$. Interesting registration results were obtained in [9] by choosing $f$ as a polynomial function. Therefore, we use a similar model for image fusion, defined as

$$
\mathbf{x}_{\mathrm{u}}=c_{1}+c_{2} \mathbf{x}_{\mathrm{m}}+c_{3}\left\|\nabla \mathbf{x}_{\mathrm{m}}\right\|_{2}
$$

where $c_{1}, c_{2}$, and $c_{3}$ are unknown polynomial coefficients.

We assume in this paper that the gradient of the high resolution MR image can be replaced by the bicubic interpolated MR image. This assumption is motivated by the fact that, as a first order approximation, the bicubic interpolated MR image $\underline{\mathbf{y}}_{\mathrm{m}} \in \mathbb{R}^{\mathbb{N}}$ contains sufficient information about the MRI contours, leading to

$$
\mathbf{x}_{\mathrm{u}}=c_{1}+c_{2} \mathbf{x}_{\mathrm{m}}+c_{3}\left\|\nabla \underline{\mathbf{y}}_{\mathrm{m}}\right\|_{2}
$$

Note that this approximation allows the US image to be written as a linear function of the MR image through (5), which will significantly simplify the following image fusion algorithm.

\subsection{Fusion of MR and US images using a Bayesian approach}

The linear model between the MR and US images in (1) yields the following fusion model

$$
\begin{aligned}
& \mathbf{y}_{\mathrm{m}}=\mathbf{S H x}+\mathbf{n}_{\mathrm{m}} \\
& \mathbf{y}_{\mathrm{u}}=c_{1}+c_{2} \mathbf{x}+c_{3}\left\|\nabla_{\mathbf{y}_{\mathrm{m}}}\right\|_{2}+\mathbf{n}_{\mathrm{u}}
\end{aligned}
$$

where $\mathbf{x} \in \mathbb{R}^{N}$ is the unknown image to be estimated, containing relevant information from both the MR and US data.

Given the noise models considered, the distributions of $\mathbf{y}_{\mathrm{m}}$ and $\mathbf{y}_{\mathbf{u}}$ are defined as

$$
\begin{aligned}
\mathbf{y}_{\mathrm{m}} \mid \mathbf{x} & \sim \mathcal{N}\left(\mathbf{S H} \mathbf{x}, \sigma_{\mathrm{m}} \mathbf{I}_{N}\right) \\
\mathbf{y}_{\mathrm{u}} \mid \mathbf{x} & \sim \mathcal{L} \mathcal{R}(\gamma)
\end{aligned}
$$

where $\mathcal{N}(\boldsymbol{\mu}, \boldsymbol{\Sigma})$ denotes the normal distribution with mean vector $\boldsymbol{\mu}$ and covariance matrix $\boldsymbol{\Sigma}$, and $\mathcal{L} \mathcal{R}(\gamma)$ is the log-Rayleigh distribution with parameter $\gamma$. Using Bayes' theorem and the independence between noises $\mathbf{n}_{\mathrm{m}}$ and $\mathbf{n}_{\mathrm{u}}$, the posterior distribution of $\mathbf{x}$ is

$$
p\left(\mathbf{x} \mid \mathbf{y}_{\mathrm{m}}, \mathbf{y}_{\mathrm{u}}\right) \propto p\left(\mathbf{y}_{\mathrm{m}} \mid \mathbf{x}\right) p\left(\mathbf{y}_{\mathrm{u}} \mid \mathbf{x}\right) p(\mathbf{x})
$$

where $p(\mathbf{x})$ is the prior probability distribution of $\mathbf{x}$ and $\propto$ means "proportional to". Thus, the log-posterior distribution can be written as

$$
\begin{aligned}
& -\log p\left(\mathbf{x} \mid \mathbf{y}_{\mathrm{m}}, \mathbf{y}_{\mathbf{u}}\right)=\underbrace{\frac{1}{2}\left\|\mathbf{y}_{\mathrm{m}}-\mathbf{S H x}\right\|_{2}^{2}}_{\text {MRI data fidelity }}+\underbrace{\log (p(\mathbf{x}))}_{\text {regularization }} \\
& +\underbrace{\sum_{i=1}^{N}\left[\exp \left(y_{\mathrm{u}, i}-f_{i}(\boldsymbol{x})\right)-\gamma\left(y_{\mathrm{u}, i}-f_{i}(\boldsymbol{x})\right)\right]}_{\text {US data fidelity }}+\mathbf{c s t}
\end{aligned}
$$

where $x_{i}$ and $f_{i}(x)=c_{1}+c_{2} x_{i}+c_{3}\left\|\nabla \underline{\boldsymbol{y}}_{\mathrm{m}}\right\|_{\mathrm{i}}$ are the $i^{\text {th }}$ pixels of $\mathbf{x}$ and $f(\mathbf{x})$, and cst is a constant.

Estimating $\mathbf{x}$ in the sense of the maximum a posteriori principle consists of minimizing the cost function in (9). In this paper, we propose to use the classical total variation (TV) to regularize the solution, in order to obtain a piece-wise constant fused image. This regularization leads to

$$
\begin{aligned}
\hat{\mathbf{x}} & =\underset{\mathbf{x}}{\operatorname{argmin}} \frac{1}{2}\left\|\mathbf{y}_{\mathrm{m}}-\mathbf{S H x}\right\|_{2}^{2} \\
& +\tau \sum_{i=1}^{N}\left[\exp \left(y_{\mathrm{u}, i}-\left(c_{1}+c_{2} x_{i}+c_{3}\left\|\nabla \underline{\boldsymbol{y}}_{\mathrm{m}}\right\|_{\mathrm{i}}\right)\right)\right. \\
& \left.-\gamma\left(y_{\mathrm{u}, i}-\left(c_{1}+c_{2} x_{i}+c_{3}\left\|\nabla \underline{\boldsymbol{y}}_{\mathrm{m}}\right\|_{\mathrm{i}}\right)\right)\right]+\frac{\lambda}{2}\|\nabla \mathbf{x}\|_{2}^{2}
\end{aligned}
$$

where $\tau$ and $\lambda$ are hyperparameters balancing the weight between US and MR data fidelity and the TV regularization terms.

\section{MR/US FUSION ALGORITHM}

To solve (10), we propose to use an algorithm based on the alternate direction method of multipliers (ADMM). ADMM is a well-known optimization algorithm that is intended to blend the decomposability of dual ascent [10]. We start by reformulating (10) with its constrained counterpart

$$
\begin{gathered}
\hat{\mathbf{x}}=\underset{\mathbf{x}_{\mathrm{mr}}}{\operatorname{argmin}} \frac{1}{2}\left\|\mathbf{y}_{\mathrm{m}}-\mathbf{S H} \mathbf{x}\right\|_{2}^{2}+\frac{\lambda}{2}\|\nabla \mathbf{x}\|_{2}^{2} \\
+\tau \sum_{i}\left[\exp \left(y_{u s, i}-v_{i}\right)-\gamma\left(y_{\mathrm{u}, i}-v_{i}\right)\right]+\frac{\lambda}{2}\|\nabla \mathbf{v}\|_{2}^{2} \\
\text { s.t. } \mathbf{v}=c_{1}+c_{2} \mathbf{x}+c_{3}\left\|\nabla \underline{\boldsymbol{y}}_{\mathrm{mr}}\right\|
\end{gathered}
$$




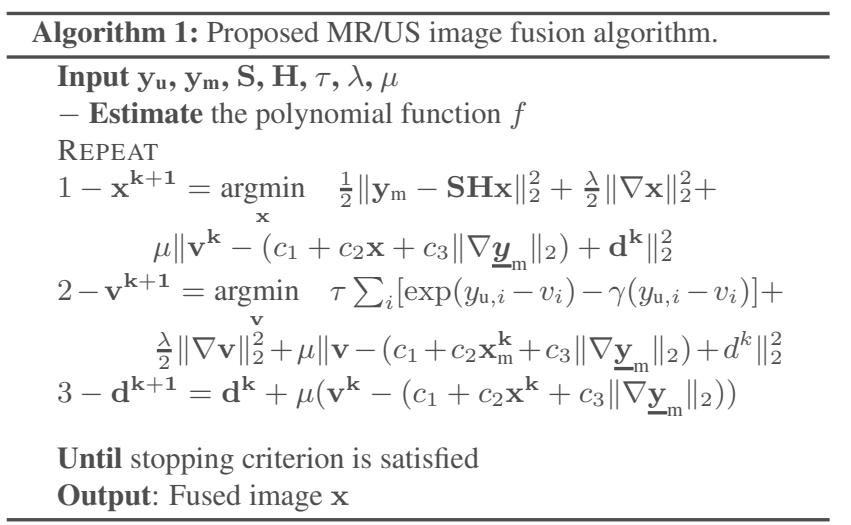

$$
\begin{aligned}
\mathcal{L}(\mathbf{x}, \mathbf{v}, \mathbf{d}) & =\frac{1}{2}\left\|\mathbf{y}_{\mathbf{m}}-\mathbf{S H x}\right\|_{2}^{2}+\frac{\lambda}{2}\|\nabla \mathbf{x}\|_{2}^{2} \\
& +\tau \sum_{i}\left[\exp \left(y_{\mathrm{u}, i}-v_{i}\right)-\gamma\left(y_{\mathrm{u}, i}-v_{i}\right)\right]+\frac{\lambda}{2}\|\nabla \mathbf{v}\|_{2}^{2} \\
& +\mu\left\|\mathbf{v}-\left(c_{1}+c_{2} \mathbf{x}+c_{3}\left\|\nabla \underline{\mathbf{y}}_{\mathrm{mr}}\right\|\right)+\mathbf{d}\right\|_{2}^{2} .
\end{aligned}
$$

Algo. 1 summarizes all the steps of the proposed MR/US image fusion method, which are detailed below.

\subsection{Update of the image $x$}

The update of $\mathbf{x}$ is achieved by minimizing the sum of quadratic functions, which admits an analytic solution computed efficiently in the Fourier domain. This computation is possible providing circulant boundary conditions and using the decomposition of the decimation matrix $\mathbf{S}$ proposed in [11]. The estimate of $\mathbf{x}$ is finally obtained as

$$
\mathbf{x}^{\mathbf{k}+\mathbf{1}}=\left[\mathbf{H}^{\mathbf{H}} \mathbf{S}^{\mathbf{H}} \mathbf{S H}+2\left(\frac{\lambda}{2} \mathbf{D}+\mu c_{2}^{2} \mathbf{I}_{\mathbf{N}}\right)\right]^{-1} \mathbf{R}
$$

where

$$
\mathbf{R}=\left(\mathbf{H}^{\mathbf{H}} \mathbf{S}^{\mathbf{H}} \mathbf{y}_{\mathrm{m}}+4 \frac{\mu}{\lambda} c_{2}\left(\mathbf{v}^{\mathbf{k}}+\mathbf{d}^{\mathbf{k}}-c_{1}-c_{3}\left\|\nabla \underline{\boldsymbol{y}}_{\mathrm{m}}\right\|\right)\right)
$$

$\mathbf{v}^{\mathrm{k}}$ and $\mathbf{d}^{\mathrm{k}}$ are estimated in the $k$-iteration, and

$$
\mathbf{D}=\mathbf{D}_{\mathbf{h}}^{\mathrm{H}} \mathbf{D}_{\mathbf{h}}+\mathbf{D}_{\mathbf{v}}^{\mathrm{H}} \mathbf{D}_{\mathbf{v}} \text {. }
$$

\subsection{Update of $v$ and $d$}

The variable $\mathbf{v}$ is updated using a gradient descent algorithm with backtracking line search, given that the function to minimize in this step is differentiable and convex. The scaled dual variable is updated analytically as mentioned in [10], see step 3 in Algo. 1.

\subsection{Estimation of the polynomial $f$}

The unknown polynomial function $f$ relating $\mathbf{x}_{\mathrm{m}}$ to $\mathbf{x}_{\mathrm{u}}$, is defined by three coefficients gathered in the vector $\mathbf{c}=\left(c_{1}, c_{2}, c_{3}\right)^{T}$. To estimate these coefficients, we propose to use the following relation

$$
y_{\mathrm{u}, i}=c_{1}+c_{2} \underline{y}_{m, i}+c_{3}\left\|\nabla \underline{\boldsymbol{y}}_{\mathrm{m}}\right\|_{i}+\epsilon_{i} \quad i=1, \ldots, N
$$

or equivalently, in algebraic form

$$
\mathbf{y}_{\mathrm{u}}=\mathbf{A c}+\epsilon
$$

where $\mathbf{A}$ is the following matrix

$$
\mathbf{A}=\left[\mathbf{1}, \mathbf{y}_{\mathrm{m}},\left\|\nabla \mathbf{y}_{\mathrm{m}}\right\|_{2}\right] .
$$

where 1 is a vector of ones.

Assuming a Gaussian error term, the maximum likelihood estimator of $\boldsymbol{c}$ (that is also the least squares estimator) is defined by $\hat{\mathbf{c}}=\mathbf{A}^{\dagger} \mathbf{y}_{\mathrm{u}}$, where the pseudo-inverse $\mathbf{A}^{\dagger}$ is computed by using the singular value decomposition of $\boldsymbol{A}$.

\subsection{Simulation setup}

This section demonstrates the efficiency of the proposed MR/US fusion method using synthetic images with controlled ground truth. More precisely, an observed MR image was generated starting from an abdominal high resolution MR image (as displayed in Fig. 1(a)). The resulting image was blurred, decimated and contaminated by an additive i.i.d. white Gaussian noise (see Fig. 2(a)). The free noise US image was generated using the polynomial relation (2), yielding the image of Fig. 1(b) and contaminated by a log-Rayleigh noise with localization parameter $\gamma$ (see Fig. 2(b)). Note that the simulated MR and US images have different gray-level intensities, resolution and noise structures. The MRI blurring kernel was a 2D-Gaussian filter of size $9 \times 9$ with variance $\sigma_{\mathrm{m}}=4$, the decimation factor was $d=4$, and $\gamma$ was equal to $10^{-3}$ in the simulation hereafter. The performance of the proposed fusion algorithm was evaluated using four quantitative metrics: the root mean square error (RMSE), the peak signal to noise ratio (PSNR), the improved signal-to-noise ratio (ISNR) and the mean structural similarity (MSSIM) defined as

$$
\begin{aligned}
\text { RMSE } & =\sqrt{\frac{1}{N}\left\|\widehat{\boldsymbol{x}}-\mathbf{x}_{\text {true }}\right\|_{2}^{2}} \text { PSNR }=20 \log _{10} \frac{\max \left(\widehat{\boldsymbol{x}}, \mathbf{x}_{\text {true }}\right)}{\operatorname{RMSE}} \\
\text { ISNR } & =10 \log _{10} \frac{\| \underline{\underline{\mathbf{y}}-\mathbf{x}_{\text {true }} \|}}{\left\|\widehat{\boldsymbol{x}}-\mathbf{x}_{\text {true }}\right\|_{2}^{2}} \\
\operatorname{MSSIM} & =\frac{1}{M} \sum_{j=1}^{M} \operatorname{SSIM}\left(\mathbf{x}_{\text {true }, \mathbf{j}}, \widehat{\mathbf{x}}_{\mathbf{j}}\right)
\end{aligned}
$$

where $\mathbf{x}_{\text {true }}$ is the ground truth, $\widehat{\boldsymbol{x}}$ is the estimated image, $\mathbf{y}$ is the bicubic interpolated MR image or its US counterpart $\mathbf{y}_{\mathrm{u}}$. M $\overline{\mathrm{SS}}$ SIM is the structural similarity implemented blockwise, $M$ is the number of local windows, $\widehat{x}_{i}$ and $x_{\text {true, }}$ are local regions extracted from $\widehat{\mathbf{x}}$ and $\mathbf{x}_{\text {true }}$ and SSIM is the structural similarity index computed for each window following its definition in [12]. Note that these metrics are used herein to compare the fused image with an estimated high resolution MR image computed using the algorithm of [11] and with an estimated denoised US image.

\section{RESULTS}

\subsection{Simulation results}

In this section, we consider the previous dataset composed of simulated MR and US images, and we solve the optimization problem (10) using the proposed ADMM-based fusion algorithm. The observed MR and US images are of sizes $64 \times 64$ and $256 \times 256$ pixels. The fused image is displayed in Fig. 2(c), illustrating the potential of the proposed algorithm to fuse information from both images. In particular, one may remark that the image in Fig. 2(c) preserves the smoothness of homogeneous tissues in MR image and enhance the tissue boundaries due to the better resolution of US image. Fig. 2(d) shows the image restored from the MR data only using a super-resolution algorithm studied in [11] with a TV regularization 


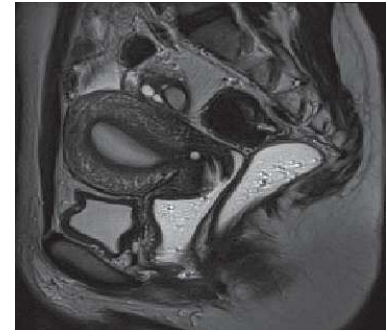

(a) Ground truth high-resolution MRI

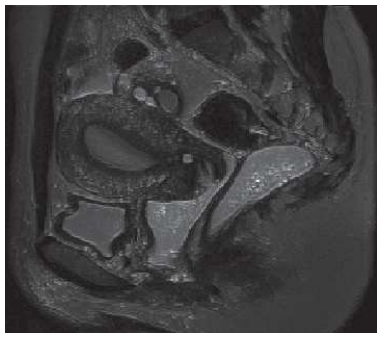

(b) Ground truth US image
Fig. 1. (a) True high-resolution MR image (b) Ground truth US image without Rayleigh noise obtained using a polynomial function applied to the MR image in (a).

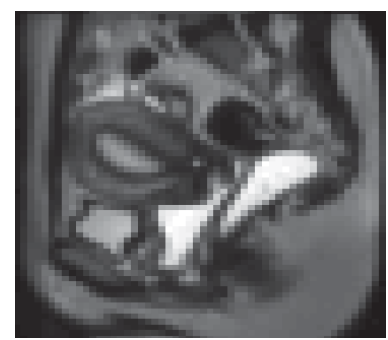

(a) MRI observation

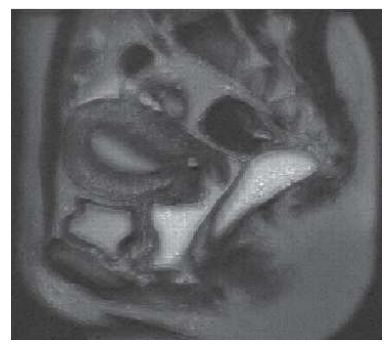

(c) Fused image

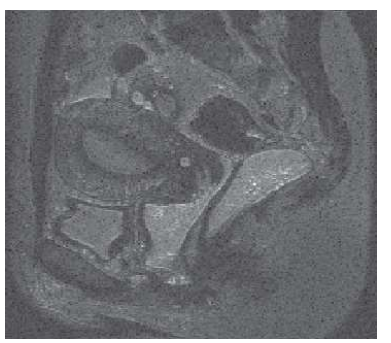

(b) US observation

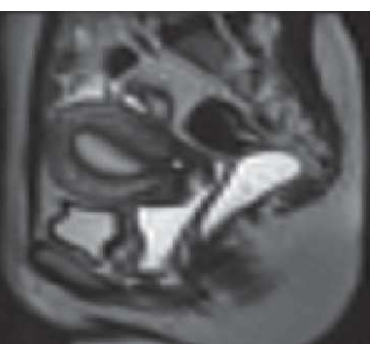

(d) Estimated HR MR image
Fig. 2. US and MRI fusion: (a) shows the MR low-resolution and blurred image, (b) shows the noisy US image, (c) shows the fused image using Algo 1, (d) shows the high resolution MR image estimated using the fast super resolution algorithm of [11].

in order to match the proposed regularization. Despite its ability to improve the quality of the low-resolution MR image, one may notice that its resolution is lower than the fused image. Note that the denoised US image, not shown in the paper because of space limitation, provides a good spatial resolution but maintains a high level of noise compared to the fused image.

The visual impression of Fig. 2 is confirmed by the quantitative results in Tab. 1, proving that the fused image obtained with the proposed method is closer to the MR ground truth than the super-resolved MR image obtained with the fast single image superresolution method of [11] and closer to the US ground truth than the denoised US image. These results confirm the importance of using both MR and US data jointly in the fusion process. Note that the peak signal to noise ratio (PSNR) and the improved signal-to-noise ratio (ISNR) are improved by more than $3 \mathrm{~dB}$ in this example.

\section{CONCLUSION}

This paper studied a new fusion method for MR and US images. The proposed method was able to reconstruct an image containing information from both images, by solving a super-resolution inverse
Table 1. Quantitative results on simulated data.

\begin{tabular}{|c|c|c|c|}
\hline & Fused image & SR MRI & Despeckled Us \\
\hline RMSE & 0.060 & 0.081 & 0.29 \\
PSNR [dB] & 24.37 & 21.08 & 10.67 \\
ISNR [dB] & 5.25 & 2.11 & 0.68 \\
MSSIM [dB] & 0.74 & 0.69 & 0.64 \\
\hline
\end{tabular}

problem for magnetic resonance images and a despeckling problem for ultrasound images. These two problems were solved jointly by using appropriate statistical models and a polynomial relationship between the images of interest. Results obtained on simulated images clearly show the advantage of combining the information provided by these two modalities rather than using them independently. Confirming the simulation results on real images is an important perspective of this preliminary work. Learning the dependency between MR and US images using machine learning methods instead of parametric models is also an interesting prospect that could provide more flexibility in the fusion model.

\section{REFERENCES}

[1] M. Amin-Naji and A. Aghagolzadeh, "Multi-focus image fusion in DCT domain using variance and energy of Laplacian and correlation coefficient for visual sensor networks," Journal of AI and Data Mining, vol. 6, pp. 233-250, 2018.

[2] M. Judenhofer and H. Wehrl, "Simultaneous PET-MRI: a new approach for functional and morphological imaging," Nature medicine, vol. 14, no. 4, p. 459, 2008.

[3] S. Gautier, G. L. Besnerais, A. Mohammad-Djafari, and L. Blandine, "Data fusion in the field of non destructive testing," in Maximum Entropy and Bayesian Methods, 1996, pp. 311-316.

[4] A. Yavariabdi and A. Bartoli, "Mapping and characterizing endometrial implants by registering $2 \mathrm{D}$ transvaginal ultrasound to 3D pelvic magnetic resonance images," Computerized Medical Imaging and Graphics, vol. 45, pp. 11-25, 2015.

[5] S. Giusti, F. Forasassi, L. Bastiani, V. Cela, N. Pluchino, V. Ferrari, E. Fruzzetti, D. Caramella, and C. Bartolozzi, "Anatomical localization of deep infiltrating endometriosis: 3D MRI reconstructions," Abdominal imaging, vol. 37, no. 6, pp. 1110-1121, 2012.

[6] F. Shi, J. Cheng, L. Wang, P.-T. Yap, and D. Shen, "LRTV: MR image super-resolution with low-rank and total variation regularizations," IEEE Trans. Med. Imaging, vol. 34, pp. 2459-2466, 2015.

[7] D. Kundu and M. Z. Raqab, "Generalized Rayleigh distribution: different methods of estimations," Computational statistics \& data analysis, vol. 49, no. 1, pp. 187-200, 2005.

[8] T. Tuthill, R. Sperry, and K. Parker, "Deviations from Rayleigh statistics in ultrasonic speckle," Ultrasonic imaging, vol. 10, no. 2, pp. 8189, 1988.

[9] A. Roche, X. Pennec, G. Malandain, and N. Ayache, "Rigid registration of 3D ultrasound with MR images: a new approach combining intensity and gradient information," IEEE Trans. Med. Imaging, vol. 20, no. 10, pp. 1038-1049, 2001.

[10] S. Boyd, N. Parikh, E. Chu, B. Peleato, J. Eckstein et al., "Distributed optimization and statistical learning via the alternating direction method of multipliers," Foundations and Trends in Machine learning, vol. 3, no. 1, pp. 1-122, 2011.

[11] N. Zhao, Q. Wei, A. Basarab, N. Dobigeon, D. Kouamé, and J.-Y. Tourneret, "Fast single image super-resolution using a new analytical solution for $l_{2}-l_{2}$ problems," IEEE Trans. Image Process., vol. 25, pp. 3683-3697, 2016.

[12] Z. Wang, A. Bovik, H. Sheikh, and E. Simoncelli, "Image quality assessment: from error visibility to structural similarity," IEEE Trans. Image Process., vol. 13, pp. 600-612, 2004. 